\title{
PONV Pressure Point Therapy
}

\author{
Joseph DiLustro* \\ Pressure Point, Inc., USA
}

Submission: December 15, 2016; Published: January 4, 2017

*Corresponding author: Joseph DiLustro, chairmen and CEO, Pressure Point, Inc., Email: therapeutics101@verizon.net

\section{News}

Pressure Point, Inc. is a global medical device company specializing in mainstream acu-point stimulation strips, which improve multi-modal anesthetic techniques associated with the complexity common problem of post-operative nausea and vomiting. The company introduced its Pressure Right@ acupressure point-stimulation product in 2011 after receiving market clearance from the FDA as a prescribed technique representing an essential part of the anesthetic plan for surgery patients. In 2014, Pressure Point subsequently received market clearance as an OTC technique for the prevention of nausea and vomiting.

As part of a multi-modal strategy for patients undergoing laparoscopic surgery, a double-blind, sham-controlled study reported that the use of Pressure Right(C) demonstrated a statistically meaningful, absolute risk reduction of PONV for surgical patients as long as 72 hours postoperatively. The study further confirmed the direct result of prophylaxis with Pressure Right(C) revealed a multi-modal PONV effect to substantially reduce the main stream requirements for expensive rescue-drug therapies. As a result, a broader patient satisfaction with PONV prevention was reported among Pressure Right $@$ study subjects.

Prior to the introduction of the multi-modal Pressure Right $($ C acu-stimulation strip in 2011 for the 72-hour prevention of PONV, a plethora of research had been published on the efficacy of acu-stimulation for PONV. Several incipient versions of acustimulation offered significant benefits and few, if any risks. However, the early product models were limited in duration for multi-modal nausea prophylaxis for surgical use and patient suitability for PONV prevention.

Outside the US today, Pressure Point @ has overseas distribution partners targeting the anesthetic market for prevention of PONV for at-risk surgical patients. Research and expanding use has proven Pressure Right $C$ to be a safe choice in PONV management. The corporate mission for the product is hope and effectiveness to bring maximum efficacy of clinical multi-modal PONV therapy to finally provide surgical patients freedom from anesthetic-related nausea and vomiting complications.

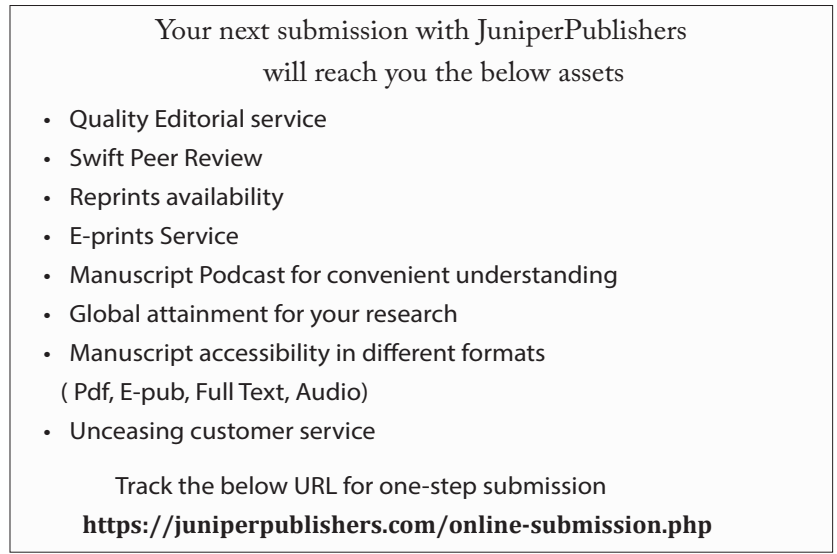

